\title{
Lipase-catalyzed Synthesis of Oleoyl- lysophosphatidylcholine by Direct Esterification in Solvent-free Medium without Water Removal
}

\author{
Taha Mnasri, Françoise Ergan, Josiane Herault and Gaëlle Pencreac'h* \\ Laboratoire Mer, Molécules, Santé (EA 2160), IUT de Laval, Université du Maine 52 rue des Drs Calmette et Guérin, BP2045, 53020 \\ Laval cedex, FRANCE
}

\begin{abstract}
In this work, the synthesis of oleoyl-lysophosphatidylcholine by lipase-catalyzed esterification of glycerophosphocholine (GPC) and free oleic acid in a reaction medium without solvent is presented. The complete solubilisation of GPC, which is a crucial issue in non-polar liquids such as melted free fatty acids, was reached by heating the GPC/oleic acid mixture at high temperature during a short time. The immobilized lipase from Rhizomucor miehei (Lipozyme RM-IM) was shown to catalyze the reaction more efficiently than the immobilized lipases from Thermomyces lanuginosus (Lipozyme TL-IM) and Candida antarctica (Novozym 435). The condition reactions leading to the highest yield were as follows: substrate ratio: $1 / 20$ (GPC/oleic acid); amount of catalyst: $10 \%$ (w/w of substrates); temperature: $50{ }^{\circ} \mathrm{C}$. Under these conditions, a yield of $75 \%$ of oleoyl-lysophosphatidylcholine was achieved in $24 \mathrm{~h}$ under stirring and almost no dioleoyl-lysophosphatidylcholine was produced. Unlike other studies dealing with the esterification of GPC with free fatty acids, the removal of the water produced while the reaction proceeds was not necessary to reach high yields.
\end{abstract}

Key words: lipase, esterification, lysophosphatidylcholine, glycerophosphocholine, oleic acid

\section{INTRODUCTION}

Lysoglycerophospholipids (or lysophospholipids, LPL) are glycerol-based bioactive lipids containing one fatty acyl moiety at either the $s n 1$ or $s n 2$ position and a phosphate group, called the head group, at the sn3 position. Except for lysophosphatidic acid (LPA), the phosphate group is esterified to an alcohol or amino-alcohol (mainly choline, ethanolamine, inositol and serine) leading to various LPL species with different head groups, such as lysophosphatidylcholine (LPC), the most abundant in nature, or lysophosphatidylethanolamine (LPE). For years, studies investigating biological activities of LPL mainly focused on $\mathrm{LPA}^{1}$. However, more recently other LPL species have also been considered as important bioactive lipids. It is now clear that many of them are involved in a large variety of both normal and pathological processes such as carcinogenesis, neurogenesis, immunity, vascular development or regulation of metabolic diseases ${ }^{2}$. Consequently it is now obvious that LPL may have therapeutic potential. Interestingly, most often, LPL effects are acyl chain-dependent, ie related to acyl-chain length and degree of unsaturation ${ }^{3-5)}$.
Moreover, LPL are important emulsifiers and wetting agents for the food-processing ${ }^{6)}$ and cosmetic $^{7}$ industries and recent studies revealed that LPL may have potential use in drug delivery ${ }^{8}$ and vaccine adjuvant ${ }^{9}$ fields.

LPL can be obtained via either chemical or enzymatic routes which are detailed in a recent review ${ }^{10)}$. Enzymatic pathways, using lipases or phospholipases, are of interest due to their stereoselective activities although building the LPL chiral structure is a crucial issue when using chemical routes. Moreover, enzymatic reactions usually occur under milder conditions, mainly lower temperatures, as compared to chemical reactions.

Most often, LPL are produced by lipase or phospholipase-catalyzed hydrolysis of natural lecithins, such as soybean, sunflower or egg yolk lecithins ${ }^{11)}$. In our laboratory, it has been shown that phospholipids extracted from microalgae, namely the prymnesiophyte Isochrysis galbana, may serve as substrate for the lipase-catalyzed production of docosahexaenoic acid-rich LPL ${ }^{12)}$. Lipasecatalyzed alcoholysis has also allowed the preparation of LPL from phospholipids ${ }^{13)}$.

\footnotetext{
*Correspondence to: Gaëlle Pencreac'h, Laboratoire Mer, Molécules, Santé (EA 2160), IUT de Laval, Université du Maine 52 rue des Drs Calmette et Guérin, BP2045, 53020 Laval cedex, FRANCE

E-mail: gaelle.pencreach@univ-lemans.fr

Accepted April 14, 2017 (received for review February 21, 2017)

Journal of Oleo Science ISSN 1345-8957 print / ISSN 1347-3352 online

http://www.jstage.jst.go.jp/browse/jos/ http://mc.manusriptcentral.com/jjocs
} 


\section{T. Mnasri, F. Ergan, J. Herault et al.}

Lipases can also catalyze ester bond synthesis in nonaqueous reaction media; however few examples of LPL synthesis using lipases can be found in the literature. They mainly describe the synthesis of LPA and LPC from glycerophosphatidic acid (GPA) and glycerophosphocholine (GPC), respectively, by either direct esterification with free fatty acids ${ }^{14-16)}$ or transesterification with fatty acid vinyl esters ${ }^{16,17)}$. However, GPA and GPC are highly polar molecules readily soluble in water and in polar solvents but nearly insoluble in the organic solvents commonly used as non-aqueous reaction media. In the literature, attempts by Kim and Kim to esterify GPC with fatty acids in hexane and isooctane were unsuccessful due to the insolubility of $\mathrm{GPC}^{14)}$. Experiments are therefore performed in solventfree systems in which the free fatty acids or esters form the liquid phase. However, GPC solubilisation in these conditions is often not complete yet and some solid GPC particles may even be visible in the reaction mixture. They slowly solubilize as the reaction proceeds ${ }^{14)}$. Similarly, GPA was reported to form a separated gel phase or to be dispersed in the media depending on the fatty acid used ${ }^{16)}$. GPA and GPC solubilisation is therefore a crucial issue for synthetic reactions with these substrates.

In ester bond synthesis reactions, another crucial parameter is the water present in the reaction medium ${ }^{18)}$. In direct esterification reaction, water is a product of the reaction which can shift the equilibrium towards hydrolysis if not removed from the reaction medium. Water is also essential to maintain the adequate hydration state of the catalyst for maximal activity. This is why, in studies dealing with the esterification of GPA or GPC, water amount is controlled by either performing the reaction under low pressure ${ }^{15)}$, adding a convenient co-solvent such as $\mathrm{DMF}^{14)}$ or equilibrating the reaction media with saturated salt solutions at specific $\mathrm{a}_{\mathrm{w}}{ }^{19)}$.

Oleoyl-LPC is one of the most important LPC species in human plasma ${ }^{20)}$ and some works have focused on elucidating its biological activities. For instance, it was shown that oleoyl-LPC containing mixed-micelles enhance the intestinal uptake of $\alpha$-tocopherol, an important dietary fat-soluble vitamin $^{21)}$. Interestingly, the counterpart phospholipid, i.e. dioleoyl-phosphatidylcholine, has the opposite effect.

In the present work, we present the synthesis of oleicLPC by direct esterification of GPC and free oleic acid. The complete solubilisation of GPC in oleic acid is achieved by stirring the mixture at high temperature during a short time. In these conditions, a high yield of reaction is reached without the need of removing the water produced during the reaction.

\section{EXPERIMENTAL}

\subsection{Chemicals}

Sn-3-glycerophosphocholine was obtained from Bachem AG (Bubendorf, Switzerland). Oleic acid (C18:1, $90 \%$ purity) was purchased from Sigma Aldrich (Saint-QuentinFallavier, France). 1-oleoyl-2-hydroxy-sn-glycero-3-phosphocholine (oleoyl-LPC) and 1,2-dioleoyl-sn-glycero3-phosphocholine (dioleoyl-PC) standards were purchased from Avanti Polar Lipids (Alabaster, USA). HPLC grade methanol was supplied from Fisher Scientific (Illkirch, France) . Lipozyme RM-IM (immobilized lipase from Rhizomucor miehei), Lipozyme TL-IM(immobilized lipase from Thermomyces lanuginosus) and Novozym 435 (immobilized lipase B from Candida antarctica) were purchased from Sigma Aldrich. All other chemicals were of analytical grade.

\subsection{Esterification reaction}

Mixtures of oleic acid $(3 \mathrm{~mL}, 9.5 \mathrm{mmol})$ with varying amounts of GPC (at GPC/oleic acid molar ratios of 1/5, 1/10, $1 / 20$ or $1 / 50$ )were prepared in $50 \mathrm{~mL}$ glass tubes by heating to $150^{\circ} \mathrm{C}$ for 15 min to reach solubility of GPC. After the reaction medium has cooled to the desired temperature, enzymatic esterification of GPC with oleic acid was initiated by addition of the lipase preparation $(1,2,5,7$ or $10 \% \mathrm{w} / \mathrm{w}$ of substrates). The reaction medium was maintained at different temperatures $\left(40^{\circ} \mathrm{C}, 50^{\circ} \mathrm{C}, 60^{\circ} \mathrm{C}\right.$ or $\left.70^{\circ} \mathrm{C}\right)$ and 750 rpm in a thermomixer $\mathrm{C}$ (Eppendorf AG, Hamburg, Germany). Samples $(50 \mu \mathrm{L})$ were withdrawn at preset time intervals and analyzed by HPLC as described below in order to quantify remaining GPC, synthesized oleoyl-LPC and dioleoyl-PC. A control without enzyme was performed for each reaction conditions.

The bioconversion yield (in \%) was defined as the concentration of formed oleoyl-LPC over the initial concentration of GPC. Results are reported as means of triplicate experiments \pm standard deviations.

\subsection{HPLC-ELSD analyses}

Samples were analyzed by reversed-phase high performance liquid chromatography (RP-HPLC) on an Agilent 1260 Infinity HPLC equipped with an evaporative light scattering detector(ELSD). Samples were diluted in methanol when necessary and $5 \mu \mathrm{L}$ of this solution were injected in the RP column(Poroshell 120 EC-18, $50 \times 4.6 \mathrm{~mm}, 2.7$ $\mu \mathrm{m})$. The components of the reaction medium were separated using the following gradient of methanol in water: $90 \%$ held for $4 \mathrm{~min}$; from 90 to $100 \%$ in $0.5 \mathrm{~min}$; held at $100 \%$ for 3.5 min; from $100 \%$ to $90 \%$ in 1 min; held at $90 \%$ for $1.5 \mathrm{~min}$. The flow rate was kept constant at 1 mL.min ${ }^{-1}$. The operating conditions for the ELSD were as follows: drift tube temperature, $40^{\circ} \mathrm{C}$; nitrogen flow, 1.6 L. $\min ^{-1}$.

All components of the reaction mixture were eluted 


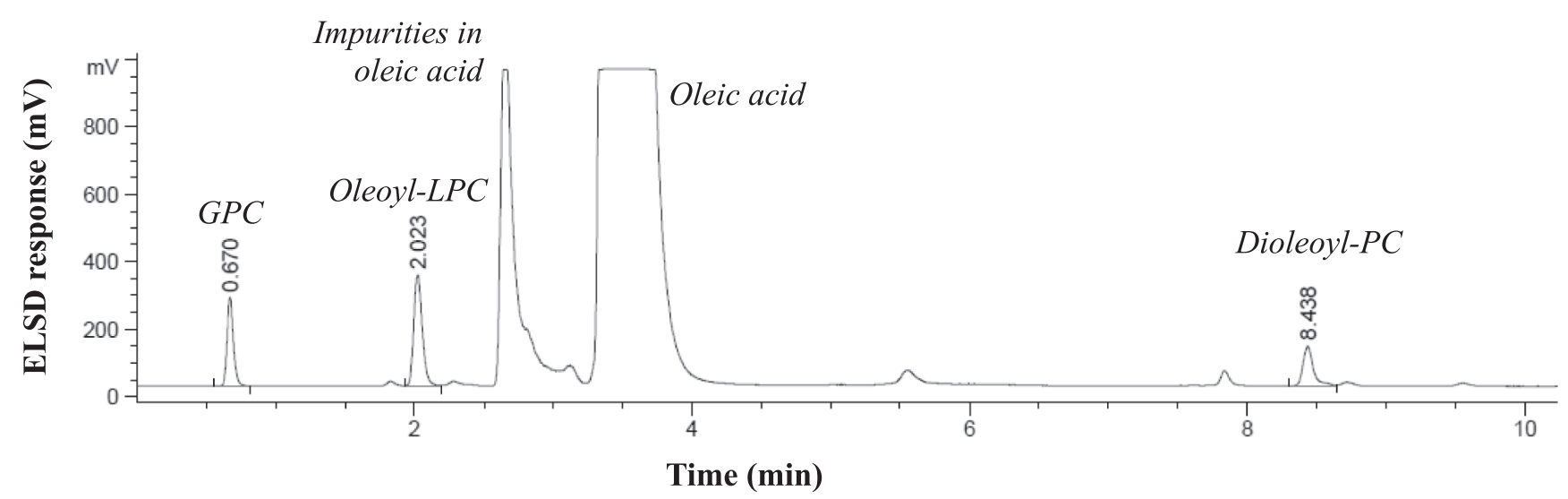

Fig. 1 RP-HPLC-ELSD chromatogram of the reaction mixture. For analysis conditions, see text paragraph 2.3.

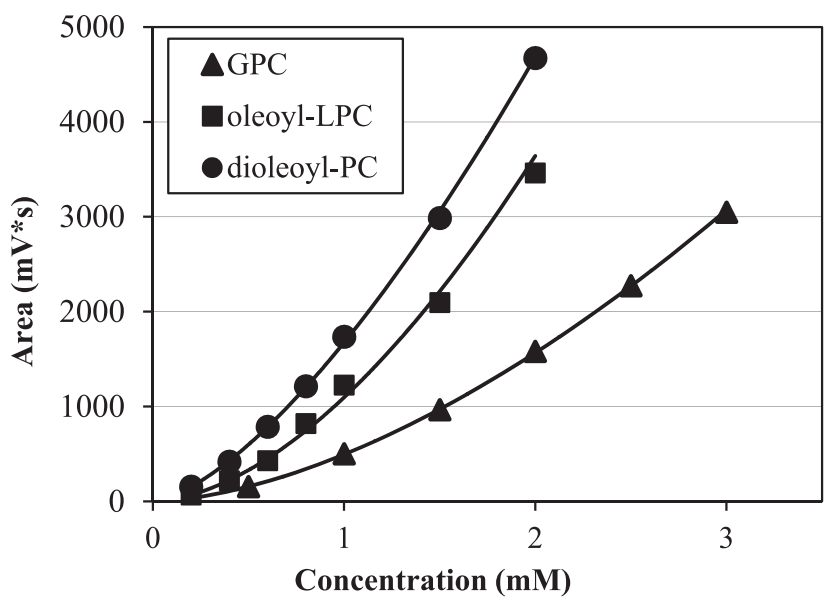

Fig. 2 Calibration curves for HPLC quantification of GPC, oleoyl-LPC and dioleoyl-PC.

within $10 \min ($ Fig. 1). The substrates GPC and oleic acid were eluted at $0.6 \mathrm{~min}$ and $3.8 \mathrm{~min}$ respectively. The two products of the reaction, oleoyl-LPC and dioleoyl-PC, were eluted at $2 \mathrm{~min}$ and $8.4 \mathrm{~min}$, respectively.

The amounts of GPC, oleoyl-LPC and dioleoyl-PC were determined using calibration curves obtained with standards (Fig. 2). The ELSD response was nonlinear and best fitted with a polynomial curve. The equations were as follows: $\mathrm{Y}=495.07 . \mathrm{X}^{1.6615}\left(\mathrm{r}^{2}=0.9999\right)$ for $\mathrm{GPC}, \mathrm{Y}=1095.7$. $\mathrm{X}^{1.7327}\left(\mathrm{r}^{2}=0.9972\right)$ for oleoyl-LPC and $\mathrm{Y}=1674.2 \cdot \mathrm{X}^{1.4849}\left(\mathrm{r}^{2}=\right.$ 0.9997) for dioleoyl-PC, with $Y$, the peak area and $X$, the concentration.

\subsection{LC-MS analysis}

Before LC-MS analysis of the reaction product, remaining oleic acid was removed by solid phase extraction using a cartridge containing $5 \mathrm{~g}$ of silica (SepPak Vac $20 \mathrm{cc}$, Waters). One $\mathrm{mL}$ of the reaction medium was loaded on the cartridge and selective elution was performed using a methanol-water step gradient as follows: $20 \mathrm{~mL} \mathrm{100 \%}$ methanol; $10 \mathrm{~mL}$ of $90 \%, 80 \%, 70 \%$ and $60 \%$ methanol.
Five $\mathrm{mL}$ fractions were collected and analyzed by HPLC. The oleoyl-LPC was desorbed with the 70 and $60 \%$ methanol eluents. The corresponding fractions were pooled and oleoyl-LPC was quantified by HPLC.

LC-MS analyses were performed at the mass spectrometry platform of the University of Maine (Institut des Molécules et Matériaux du Mans, France) using an UHPLC U3000 (Thermo Scientific, USA) coupled to a MicroOTOF-Q III mass spectrometer(Brucker, Germany) equipped with an electrospray(ESI)ion source. UV-detection at $210 \mathrm{~nm}$ was also performed. A Nucleodur C18 Htec column $(250 \times$ $2 \mathrm{~mm}, 5 \mu \mathrm{m}$ ) (Marcherey-Nagel Eurl, France)heated at $30^{\circ} \mathrm{C}$ and a mobile phase consisting of methanol (solvent A) and formic acid $0.1 \%$ in water (solvent B) at a constant flow rate of $0.2 \mathrm{~mL} \cdot \mathrm{min}^{-1}$ were used for LC separation. A $30 \mathrm{~min}$ elution gradient was applied as follows: from $80 \%$ to $100 \%$ of solvent A in $20 \mathrm{~min}$; held at $100 \%$ for $5 \mathrm{~min}$; from $100 \%$ to $80 \%$ in $1 \mathrm{~min}$; held at $80 \%$ for $4 \mathrm{~min}$. The analysed sample volume was $10 \mu \mathrm{L}$.

Samples were diluted to approximatively $10 \mu \mathrm{g} \cdot \mathrm{mL}^{-1}$ in methanol prior to analysis. ESI analyses were performed in the positive mode with a spray voltage of $+4.5 \mathrm{kV}$.

\section{RESULTS AND DISCUSSION}

\subsection{Solubilisation of GPC in oleic acid}

In our work, full GPC solubilisation in oleic acid before the reaction starts is achieved by heating the mixture at high temperature. Figure 3 shows the effect of the temperature on the time required to solubilize $16 \mathrm{mg}$ of $\mathrm{GPC}$ (i.e. $62.2 \mu \mathrm{mol})$ in $1 \mathrm{~mL}$ of oleic acid, under stirring. These amounts correspond to a molar ratio of substrates of $1 / 50$ (GPC/oleic acid). Solubilisation was completed when GPC particles were no more visible in the mixture. As seen in Fig. 3, the time required for GPC solubilisation drastically decreases from $18 \mathrm{~h}$ down to $2 \mathrm{~h}$ when the mixture temperature was increased from 60 to $80^{\circ} \mathrm{C}$. However, for higher GPC amounts, $80^{\circ} \mathrm{C}$ is no longer sufficient to ensure 


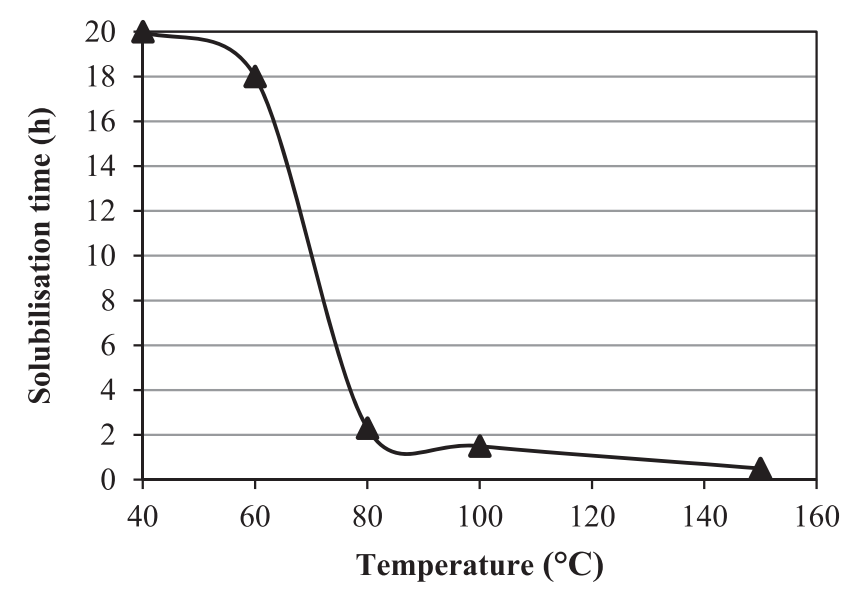

Fig. 3 Solubilisation time of GPC in oleic acid at a function of temperature. GPC (16 mg) was mixed with $1 \mathrm{~mL}$ of oleic acid and incubated at 40, 60, 80, 100 and $150^{\circ} \mathrm{C}$ until complete solubilisation.

solubilisation in a short time. It was determined that a temperature of $150^{\circ} \mathrm{C}$ allows the solubilisation of GPC within 15 min for all concentrations tested in this work.

It should be noticed that no GPC aggregation appeared after cooling the mixture to reaction temperature. Moreover no pellet was observed after centrifugation at $2000 \mathrm{~g}$ during 15 min. Finally, for all GPC amounts tested, the GPC concentration, determined by HPLC, showed no significant variation before and after centrifugation and was equal to the expected concentration.

Importantly, HPLC chromatogram of oleic acid after heating at $150^{\circ} \mathrm{C}$ for 15 min revealed neither additional peaks nor modification of retention times compared to non-heated oleic acid, proving that the proposed protocol for GPC solubilisation did not cause oleic acid denaturation.

Moreover it was checked by HPLC analysis that the heattreatment did not give rise to non-enzymatic esterification of GPC and oleic acid.

\subsection{Selection of the lipase}

Lipozyme RM-IM, Lipozyme TL-IM and Novozym 435, three immobilized lipases, were compared for their ability to produce oleoyl-LPC. The reaction conditions were set as follows: enzyme loading: $5 \% \mathrm{w} / \mathrm{w}$ of total substrate weight, molar ratio of substrates: 1/20 (GPC/oleic acid), temperature: $40^{\circ} \mathrm{C}$, agitation speed: $750 \mathrm{rpm}$. Figure 4 shows that Lipozyme RM-IM is the most effective enzyme preparation in these conditions, leading to $56 \%$ of the initial GPC esterified after $24 \mathrm{~h}$ of reaction. Interestingly, no dioleoyl-PC was detected. The two other lipase preparations give much lower yields: $32 \%$ for Lipozyme TL-IM and 8\% for Novozym 435. A control without enzyme shows that nonenzymatic reaction did not occur. Therefore, Lipozyme RM-IM will be used for further experiments.

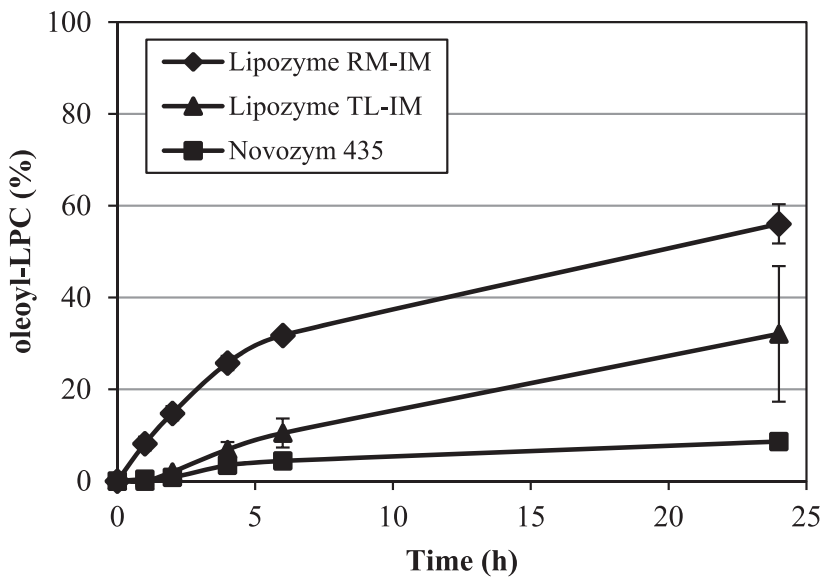

Fig. 4 Lipase screening for the esterification of GPC and oleic acid. Reaction conditions: lipase amount: 5\% (w/w substrate weight), substrate molar ratio: 1/20 (GPC/oleic acid), temperature: $40^{\circ} \mathrm{C}$, agitation speed: $750 \mathrm{rpm}$. Values are means of three replicates $\pm \mathrm{SD}$.

Although the reaction yields from different studies can be hardly compared because of great differences in reaction conditions used, it is clear from the present work and the literature that immobilized lipases are suitable catalysts for the synthesis of LPC by direct esterification of GPC with fatty acids. Kim and $\mathrm{Kim}^{22)}$ compared five lipases for the esterification of GPC with palmitic acid, two of them being immobilized lipases (Lipozyme TL-IM and Novozym 435) and three being free lipases. Lipozyme RM-IM and Novozym 435 allowed respectively $68 \%$ and $42 \%$ of GPC conversion to LPC. Among the free lipases, Aspergillus lipase (Lipase AP-6) offered similar activity as immobilized lipases (50\%) although the other two displayed the lowest activities (34-36\%). Hong et $a l .{ }^{15)}$ compared three immobilized lipases (Lipozyme RM-IM, Lipozyme TL-IM and Novozym 435) and two free phospholipases (PLA1 Lecitase Ultra and PLA2 Lecitase 10L) for the esterification of GPC with conjugated linoleic acids. Interestingly, the two phospholipases are much less effective $(<10 \%$ GPC conversion) than the immobilized lipases for the production of LPC. The highest yield for LPC was obtained using Novozym 435.

\subsection{Optimisation of reaction conditions}

\subsubsection{Lipase load}

The effect of Lipozyme RM-IM amount in the reaction medium was studied using 1, 2, 5, 7 and $10 \%$ of enzyme (w/ $\mathrm{w}$ of substrates). The molar ratio of substrates, the reaction temperature and the agitation speed were kept similar as in 3.2 , namely $1 / 20,40^{\circ} \mathrm{C}$ and $750 \mathrm{rpm}$ respectively. Produced oleoyl-LPC and dioleoyl-PC were quantified after 96 $\mathrm{h}$ of reaction. Figure $5 \mathrm{~A}$ shows that higher amounts of enzyme allow higher production of oleoyl-LPC. In the same 

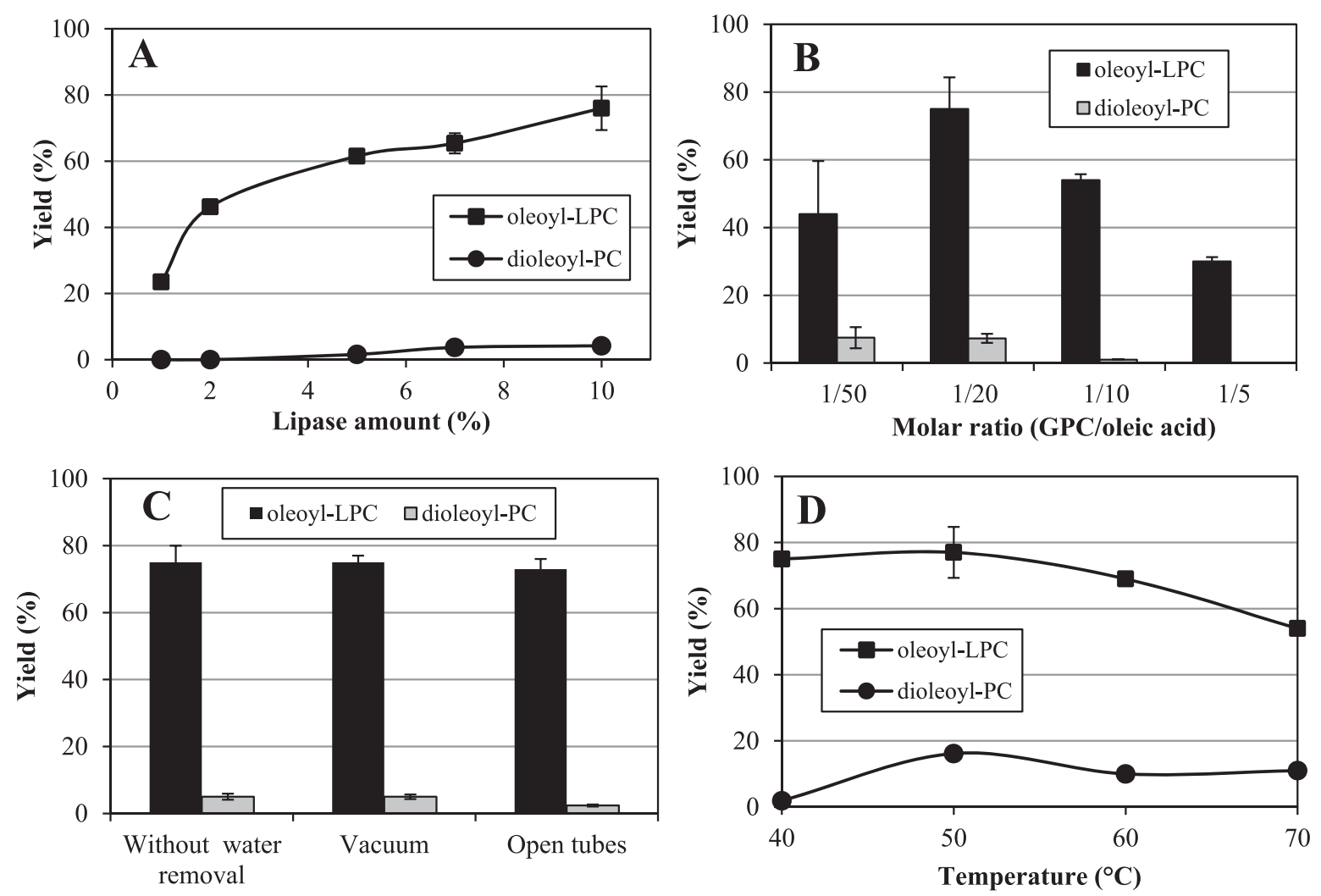

Fig. 5 Effect of reaction conditions on the esterification yield of GPC and oleic acid with Lipozyme RM-IM. A) Lipase amount (substrate molar ratio: 1/20 (GPC/oleic acid), 40 C, $750 \mathrm{rpm}, 96 \mathrm{~h}$ ); B) Substrate molar ratio (GPC/oleic acid) (lipase amount: 10\% (w/w substrate weight), 40 $, 750 \mathrm{rpm}, 72 \mathrm{~h}$ ); C) Water removal(lipase amount: $10 \%$ (w/w substrate weight), substrate molar ratio: $1 / 20$ (GPC/oleic acid), $40^{\circ} \mathrm{C}, 750 \mathrm{rpm}, 72 \mathrm{~h}$ ); D) Temperature (lipase amount: 10\% (w/w substrate weight), substrate molar ratio: 1/20 (GPC/oleic acid), $750 \mathrm{rpm}, 72 \mathrm{~h}$ ). Values are means of three replicates $\pm \mathrm{SD}$.

time, very little dioleoyl-PC is produced whatever the enzyme amount used.

Reaction equilibrium is not reached in $96 \mathrm{~h}$ with amounts of enzyme lower than $10 \%$. The confirmation that the yield obtained with $10 \%$ of enzyme actually corresponds to the reaction equilibrium, would have required to test an even higher amount of enzyme. However, such amounts lead to a very thick reaction medium where stirring becomes impossible. For this reason, the enzyme amount will be kept at $10 \%$ (w/w of substrates) for further experiments. It has been checked that initial rates were proportional to enzyme amounts (data not shown).

\subsubsection{GPC/oleic acid molar ratio}

Molar ratios of substrates (GPC/oleic acid) varying from $1 / 50$ to $1 / 5$ have been tested at $40^{\circ} \mathrm{C}$. The highest production of oleoyl-LPC is obtained with a ratio of $1 / 20$ (Fig. 5B). $75 \%$ of oleoyl-LPC is obtained after $72 \mathrm{~h}$ of reaction while the other substrate ratios lead to yields between 30 and $50 \%$. In all cases, dioleoyl-PC remains below $10 \%$. No reaction occurred without enzyme in any of the studied conditions.

The lower yields obtained with higher substrate ratios can be due to the important viscosity of the medium since increasing the amount of GPC actually increases the viscosity of the reaction medium significantly.

\subsubsection{Removal of produced water}

In an attempt to increase reaction yields, the water produced during the reaction has been eliminated from the reaction medium by evaporation either by maintaining the reaction tubes open or by performing the reaction under vacuum at 20 mbar (Multivapor ${ }^{\circledR}$ P-6 system, Buchi, Switzerland). In both cases, no increase of oleoyl-LPC and dioleoyl-PC amounts is observed after $72 \mathrm{~h}$ of reaction with respect to the experiment without water removal (Fig. 5C).

In literature, water present in the reaction medium is often a key parameter that has to be carefully controlled to reach high reaction yields. Kim and $\mathrm{Kim}^{14)}$ have obtained a 2.5 -fold increase (from 25 to $62 \%$ ) of the esterification yield of GPC and oleic acid when $4 \%$ of DMF was added to the reaction medium. Interestingly they showed that it was due to the ability of DMF to decrease water activity of the reaction medium and to deprive the enzyme of water. In the same manner, Hong et al. ${ }^{15)}$ have applied vacuum to remove the water produced during esterification of GPC with con- 
jugated linoleic acids. The initial yield of a few percents increased up to $70 \%$ when applying a vacuum of $1 \mathrm{~mm} \mathrm{Hg}$. Salt hydrate pairs have been used by Han and Rhee ${ }^{19)}$ to improve the reaction yield from $16 \%$ up to $36 \%$ with capric acid.

In our conditions, it was shown that the water produced during the esterification does not lead to consequent hydrolysis of produced LPL or PL that would limit the yield of the esterification reaction.

Regarding the initial water activity $\left(\mathrm{a}_{\mathrm{w}}\right)$ of the reaction mixture(AqualabLite ${ }^{\circledast}$ apparatus, Decagon Devices Inc., USA), no significant modification arose after the heattreatment at $150^{\circ} \mathrm{C}$ for 15 min necessary for GPC solubilisation. Indeed, $\mathrm{a}_{\mathrm{w}}$ of GPC/oleic acid mixture were 0.28 and 0.21 , before and after heating followed by cooling to room temperature, respectively.

The lack of water removal impact on reaction yield could therefore be related to the complete solubilisation of GPC. Indeed, if GPC is not well solubilized, the mass transfer of GPC from solid particles to the micro-environment of the enzyme could limit the reaction.

\subsubsection{Reaction temperature}

Figure 5D shows oleoyl-LPC and dioleoyl-PC yields obtained after $72 \mathrm{~h}$ of reaction at different temperatures ranging from $40^{\circ} \mathrm{C}$ to $70^{\circ} \mathrm{C}$. Because of the low solubility of GPC in oleic acid, it is not possible to work below $40^{\circ} \mathrm{C}$. Increasing the temperature does not change significantly the production of dioleoyl-PC. Oleoyl-LPC production is not increased by increasing the temperature to $50^{\circ} \mathrm{C}$. However the oleoyl-LPC yield decreases with increasing temperatures over $50^{\circ} \mathrm{C}$. None of the studied temperatures led to non-enzymatic synthesis of oleoyl LPC.

Nevertheless, the $75 \%$ yield is reached in only $24 \mathrm{~h}$ when working at $50^{\circ} \mathrm{C}$ rather than in $72 \mathrm{~h}$ when the esterification is carried out at $40^{\circ} \mathrm{C}$ (data not shown). For this reason, $50^{\circ} \mathrm{C}$ is determined as being optimal for this reaction.

\subsection{Structural analysis of synthesized oleoyl-LPC by LC- MS}

In order to structurally analyse the product of the reaction, the exact mass and the acyl group position were determined by LC-MS. The conditions used for LC separation allowed the separation of the $s n 1$ and $s n 2$ isomers of oleoyl-LPC, as proved by the analysis of a standard solution of sn 1 -oleoyl-LPC containing up to $10 \%$ of the sn 2 isomer (data from the supplier). The $s n 2$ isomer is first eluted at $18.5 \mathrm{~min}$ and the $\operatorname{sn} 1$ isomer is then eluted at $19.6 \mathrm{~min}$. Peak areas represent $6.6 \%$ and $93.4 \%$ of total area, respectively.

LC chromatogram of the purified product displays one major peak eluted at $19.3 \mathrm{~min}$ and a minor peak eluted at 18.4 min. For both peaks, corresponding ESI positive-ion mass spectrum displays two main ions at $m / z 522$ and 544 , corresponding to theoretical masses of the protonated ion $\left([\mathrm{M}+\mathrm{H}]^{+}\right)$and the sodium adduct ion $\left([\mathrm{M}+\mathrm{Na}]^{+}\right)$of oleoyl-LPC, respectively. They therefore correspond to sn 1 oleoyl-LPC and $s n 2$-oleoyl-LPC respectively. Peak areas represent $97 \%$ and $3 \%$ of total area, respectively.

The lipase from Rhizomucor miehei, in a free or immobilized form, is a well-known industrial biocatalyst used in a wide range of applications. It displays a 1,3-regioselectivity towards triacylglycerols both for hydrolysis and esterification $^{23)}$. Regarding the esterification of GPC with oleic acid, it preferentially catalyses the acylation of the $s n 1$ position as expected. The same observation was made by Virto et al. ${ }^{16)}$ using the immobilized lipase from Rhizopus arrhizus for the catalysis of the esterification of GPA and vinyl laurate.

\subsection{Kinetics of the reaction}

Experimental conditions previously established were used to follow the esterification of GPC with oleic acid as a function of time: $10 \%(\mathrm{w} / \mathrm{w})$ Lipozyme RM-IM, molar ratio GPC/oleic acid 1/20 without water removal and at $50^{\circ} \mathrm{C}$. GPC decreases sharply for the first hours while oleoyl-LPC increases up to $75 \%$ in $20 \mathrm{~h}$ and no dioleoyl-PC is produced (Fig. 6). Nevertheless, after $20 \mathrm{~h}$ of reaction, dioleoyl-PC starts to be slowly produced and goes up to $15 \%$ in $72 \mathrm{~h}$. As suggested by Virto et al. ${ }^{16)}$ the production of dioleoyl-PC might result from the acylation of the $s n 1$ position after spontaneous intramolecular migration of the acyl group from the $s n 1$ to the $s n 2$ position in oleoyl-LPC. However the $s n 1$ isomer being more stable than the $s n 2$ isomer, this phenomena occurs slowly ${ }^{24,25)}$ explaining the very low production of dioleoyl-PC.

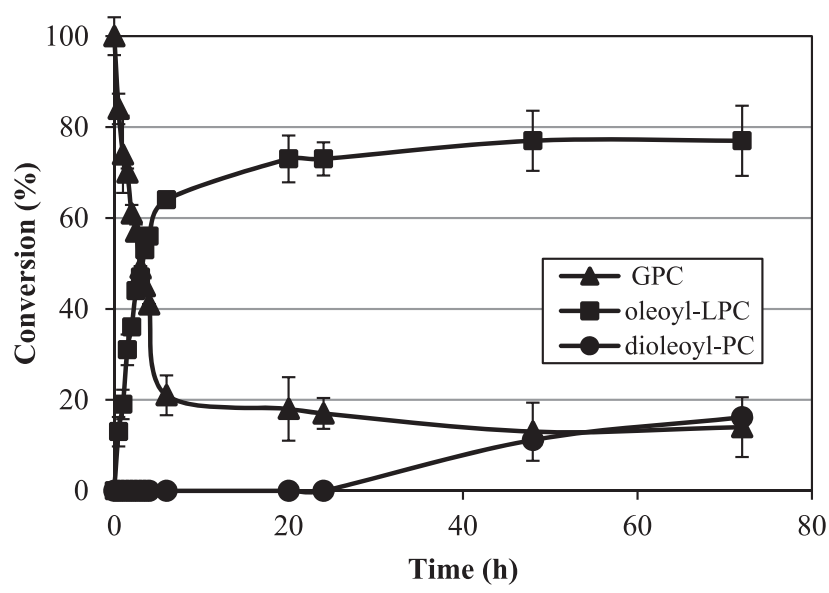

Fig. 6 Kinetics of the esterification of GPC and oleic acid catalyzed by Lipozyme RM-IM. Reaction conditions: lipase amount: $10 \%(\mathrm{w} / \mathrm{w}$ substrate weight), substrate molar ratio: 1/20 (GPC/oleic acid), temperature: $50^{\circ} \mathrm{C}$, agitation speed: $750 \mathrm{rpm}$, no water removal. Values are means of three replicates \pm SD. 


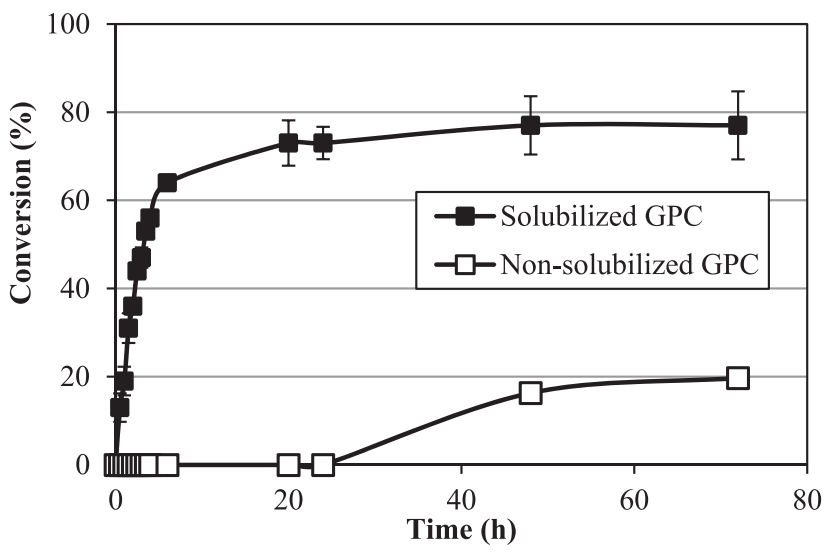

Fig. 7 Comparison of esterification of GPC and oleic acid catalyzed by Lipozyme RM-IM with solubilized GPC and non-solubilized GPC. Reaction conditions: lipase amount: 10\%(w/w substrate weight), substrate molar ratio: 1/20 (GPC/oleic acid), temperature: $50^{\circ} \mathrm{C}$, agitation speed: $750 \mathrm{rpm}$, no water removal. GPC was solubilised by heating GPC/oleic acid mixture at $150^{\circ} \mathrm{C}$ for $15 \mathrm{~min}$. With non-solubilized GPC, GPC concentrations could not be determined accurately. Conversion was therefore calculated using the theoretical initial GPC concentration instead of the measured initial concentration.

In order to confirm that complete solubilisation of GPC represents a crucial issue to reach high reaction yields, the reaction was performed omitting the preliminary $150^{\circ} \mathrm{C}$ -treatment of GPC/oleic acid mixture. The substrate mixture was agitated at the reaction temperature, i.e. $50^{\circ} \mathrm{C}$, for $15 \mathrm{~min}$ before the reaction was started by enzyme addition. In these conditions, no oleoyl-LPC was detected in the reaction medium in the first $24 \mathrm{~h}$ of reaction and the reaction yield after $72 \mathrm{~h}$ of reaction reached only $20 \%$ (Fig. 7).

\section{CONCLUSION}

Enzymatic esterification of oleic acid and GPC in a solvent-free medium with Lipozyme RM-IM has been achieved. A yield of $75 \%$ of oleoyl-LPC is reached in $24 \mathrm{~h}$ at $50^{\circ} \mathrm{C}$ or $72 \mathrm{~h}$ at $40^{\circ} \mathrm{C}$ with a molar ratio of $1 / 20$ (GPC/oleic acid) and an enzyme load of $10 \%$ (w/w of substrates). No dioleoyl-PC was produced within the first $24 \mathrm{~h}$ of reaction.

Unlike in other studies from the literature, complete solubilisation of GPC in the liquid fatty acid before the reaction starts was achieved. This allows the GPC concentration in the close environment of the enzyme to be higher than when GPC is initially under the form of solid particles and dissolves slowly as the reaction proceeds. Therefore the equilibrium of the reaction is in favour of the ester bond synthesis reaction and no water removal was necessary to reach a $75 \%$ yield of oleoyl-LPC in $24 \mathrm{~h}$.

\section{Acknowledgments}

This research was financed by the "Conseil Départemental de la Mayenne" and "Laval Agglomération". The authors greatly acknowledge Emmanuelle Mebold and Patricia Gangnery for mass spectrometry analyses.

\section{References}

1) Lin, M.-E.; Herr, D.R.; Chun, J. Lysophosphatidic acid (LPA) receptors: signaling properties and disease relevance. Prostaglandins Other Lipid Mediat. 91, 130138 (2010).

2) Grzelczyk, A.; Gendaszewska-Darmach, E. Novel bioactive glycerol-based lysophospholipids: New data New insight into their function. Biochimie 95, 667679 (2013).

3) Riederer, M.; Ojala, P.J.; Hrzenjak, A.; Graier, W.F.; Malli, R.; Tritscher, M.; Hermansson, M.; Watzer, B.; Schweer, H.; Desoye, G.; Heinemann, A.; Frank, S. Acyl chain-dependent effect of lysophosphatidylcholine on endothelial prostacyclin production. J. Lipid Res. 51, 2957-2966 (2010).

4) Brkić, L.; Riederer, M.; Graier, W. F.; Malli, R.; Frank, S. Acyl chain-dependent effect of lysophosphatidylcholine on cyclooxygenase (COX) -2 expression in endothelial cells. Atherosclerosis 224, 348-354(2012).

5) Rao, S.P.; Riederer, M.; Lechleitner, M.; Hermansson, M.; Desoye, G.; Hallström, S.; Graier, W.F.; Frank, S. Acyl chain-dependent effect of lysophosphatidylcholine on endothelium-dependent vasorelaxation. PLoS ONE 8, e65155 (2013).

6) Kasinos, M.; Goñi, M.L.; Nguyen, M.T.; Sabatino, P.; Martins, J.C.; Dewettinck, K.; Van der Meeren, P. Effect of hydrolysed sunflower lecithin on the heat-induced coagulation of recombined concentrated milk emulsions. Int. Dairy J. 38, 187-194(2014).

7) Yahagi, S.; Koike, M.; Okano, Y.; Masaki, H. Lysophospholipids improve skin moisturization by modulating of calcium-dependent cell differentiation pathway. Int. J. Cosmet. Sci. 33, 251-256 (2011).

8) Koklic, T.; Trancar, J. Lysolipid containing liposomes for transendothelial drug delivery. BMC Res. Notes 5, 179 (2012).

9) Cmielewski, P.; Anson, D.S.; Parsons, D.W. Lysophosphatidylcholine as an adjuvant for lentiviral vector mediated gene transfer to airway epithelium: effect of acyl chain length. Respir. Res. 11, 84(2010).

10) D’Arrigo, P.; Servi, S. Synthesis of lysophospholipids. 
Molecules 15, 1354-1377 (2010).

11) Cabezas, D.M.; Madoery, R.; Diehl, B.W.K.; Tomás, M.C. Emulsifying properties of different modified sunflower lecithins. J. Am. Oil Chem. Soc. 89, 355-361 (2012).

12) Devos, M.; Poisson, L.; Ergan, F.; Pencreac'h, G. Enzymatic hydrolysis of phospholipids from Isochrysis galbana for docosahexaenoic acid enrichment. Enzyme Microb. Technol. 39, 548-554(2006).

13) Yang, G.; Yang, R.; Hu, H. Lysophosphatidylcholine synthesis by lipase-catalyzed ethanolysis. J. Oleo Sci. 64, 443-447 (2015).

14) Kim, J.; Kim, B.G. Lipase-catalyzed synthesis of lysophosphatidylcholine using organic cosolvent for in situ water activity control. J. Am. Oil Chem. Soc. 77, 791797 (2000).

15) Hong, S.I.; Kim, Y.; Kim, C.T.; Kim, I.H. Enzymatic synthesis of lysophosphatidylcholine containing CLA from sn-glycero-3-phosphatidylcholine (GPC) under vacuum. Food Chem. 129, 1-6 (2011).

16) Virto, C.; Svensson, I.; Adlercreutz, P. Enzymatic synthesis of lysophosphatidic acid and phosphatidic acid. Enzyme Microb. Technol. 24, 651-658(1999).

17) Virto, C.; Adlercreutz, P. Lysophosphatidylcholine synthesis with Candida antarctica lipase B (Novozym 435). Enzyme Microb. Technol. 26, 630-635 (2000).

18) Stergiou, P.Y.; Foukis, A.; Filippou, M.; Koukouritaki, M.; Parapouli, M.; Theodorou, L.G.; Hatziloukas, E.; Afendra, A.; Pandey, A.; Papamichael, E.M. Advances in lipase-catalyzed esterification reactions. Biotech- nol. Adv. 31, 1846-1859(2013).

19) Han, J.J.; Rhee, J.S. Effect of salt hydrate pairs for water activity control on lipase-catalyzed synthesis of lysophospholipids in a solvent-free system. Enzyme Microb. Technol. 22, 158-164(1998).

20) Ojala, P.J.; Hirvonen, T.E.; Hermansson, M.; Somerharju, P.; Parkkinen, J. Acyl chain-dependent effect of lysophosphatidylcholine on human neutrophils. J. Leukoc. Biol. 82, 1501-1509(2007).

21) Koo, S.I.; Noh, S.K. Phosphatidylcholine inhibits and lysophosphatidylcholine enhances the lymphatic absorption of $\alpha$-tocopherol in adult rats. J. Nutr. 131, 717-722 (2001).

22) Kim, J.; Kim, B.G. Lipase-catalyzed synthesis of lysophosphatidylcholine. Ann. N. Y. Acad. Sci. 864, 341344 (1998).

23) Rodrigues, R.C.; Fernandez-Lafuente, R. Lipase from Rhizomucor miehei as a biocatalyst in fats and oils modification. J. Mol. Catal. B Enzym. 66, 15-32 (2010).

24) Poisson, L.; Devos, M.; Godet, S.; Ergan, F.; Pencreac'h, G. Acyl migration during deacylation of phospholipids rich in docosahexaenoic acid (DHA): an enzymatic approach for evidence and study. Biotechnol. Lett. 31, 743-749 (2009).

25) Li, W.; Du, W.; Li, Q.; Sun, T.; Liu, D. Study on acyl migration kinetics of partial glycerides: Dependence on temperature and water activity. J. Mol. Catal. B Enzym. 63, 17-22(2010). 